\title{
Enhanced effect and mechanism of water-in-oil microemulsion as an oral delivery system of hydroxysafflor yellow $A$
}

This article was published in the following Dove Press journal:

International Journal of Nanomedicine

9 May 201I

Number of times this article has been viewed

\author{
Jianping $Q i^{1,2}$ \\ Jie Zhuang' \\ Wei Wu ${ }^{2}$ \\ Yi Lu ${ }^{2}$ \\ Yunmei Song ${ }^{3}$ \\ Zhetao Zhang' \\ Jia Jia' \\ Qineng Ping'
}

'School of Pharmacy, China Pharmaceutical University, Nanjing, People's Republic of China; ${ }^{2}$ School of Pharmacy, Fudan University,

Shanghai, People's Republic of China;

${ }^{3}$ School of Pharmacy and Medical

Sciences, University of South

Australia, Adelaide, Australia
Correspondence: Qineng Ping

School of Pharmacy,

China Pharmaceutical University,

24 Tong Jia Xiang, Nanjing 210009 ,

People's Republic of China

Tel +1862583271098

Fax +1862583301606

Email pingqineng2004@yahoo.com.cn
Background: A microemulsion is an effective formulation for improving the oral bioavailability of poorly soluble drugs. In this paper, a water-in-oil (w/o) microemulsion was investigated as a system for enhancing the oral bioavailability of Biopharmaceutic Classification System (BCS) III drugs.

Methods: The microemulsion formulation was optimized using a pseudoternary phase diagram, comprising propylene glycol dicaprylocaprate (PG), Cremophor ${ }^{\circledR}$ RH40, and water (30/46/24 w/w).

Results: The microemulsion increased the oral bioavailability of hydroxysafflor yellow A which was highly water-soluble but very poorly permeable. The relative bioavailability of hydroxysafflor yellow A microemulsion was about 1937\% compared with a control solution in bile duct-nonligated rats. However, the microemulsion showed lower enhanced absorption ability in bile duct-ligated rats, and the relative bioavailability was only $181 \%$. In vitro experiments were further employed to study the mechanism of the enhanced effect of the microemulsion. In vitro lipolysis showed that the microemulsion was digested very quickly by pancreatic lipase. About $60 \%$ of the microemulsion was digested within 1 hour. Furthermore, the particle size of the microemulsion after digestion was very small $(53.3 \mathrm{~nm})$ and the digested microemulsion had high physical stability. An everted gut sac model demonstrated that cumulative transport of the digested microemulsion was significantly higher than that of the diluted microemulsion.

Conclusion: These results suggested that digestion of the microemulsion by pancreatic lipase plays an important role in enhancing oral bioavailability of water-soluble drugs.

Keywords: propylene glycol dicaprylocaprate, microemulsion, hydroxysafflor yellow A, bioavailability, lipolysis

\section{Introduction}

Lipid-based formulations have been widely used as oral delivery systems for poorly soluble drugs, including microemulsions, oil suspensions, and self-emulsifying systems. ${ }^{1-3}$ Oral lipid-based formulations have been marketed for over two decades, and presently comprise an estimated $2 \%-4 \%$ of commercially available drug products, according to a survey in three markets worldwide. ${ }^{4}$ Lipid-based formulations can increase the bioavailability of poorly soluble drugs. The mechanisms of absorption enhancement are very complicated, involving improved solubility of drug molecules, formation of mixed micelles, opening of tight junctions, and improved lymphatic delivery. ${ }^{5,6}$ Microemulsions also have the potential to increase the permeability of hydrophilic peptides and proteins across the intestinal mucosa, thus enhancing their bioavailability. ${ }^{7,8}$ One of the proposed mechanisms involves enhancer-induced structural 
and fluidity changes in the mucosal membrane. ${ }^{9}$ However, there are few reports on the enhancing effect of water-in-oil (w/o) microemulsions on Biopharmaceutics Classification System (BCS) III low molecular weight drugs. This study was undertaken to confirm this effect, using hydroxysafflor yellow A (HSYA) as a model drug.

HSYA is extracted from the flower of the safflower plant, Carthamus tinctorius L. HSYA is the active ingredient of the safflower plant, and has been demonstrated to antagonize platelet-activating factor receptor binding, and thus is used to treat several ischemic diseases, including myocardial ischemia, cerebral ischemia, coronary heart disease, and cerebral thrombosis. ${ }^{10-12}$ According to recent studies, HSYA is a hydrophilic drug with low oral bioavailability, belonging to the BCS III class of drugs. ${ }^{13}$ Our previous studies have suggested that medium chain triglycerides, and chuanxiong volatile oil ${ }^{14}$ could increase the bioavailability of HSYA. However, the solubility of HSYA in oil is very low, so the compound must be prepared as a phytosome for increasing its solubility in oil. w/o microemulsion can dissolve water-soluble molecules in the inner phase, so may be the optimal formulation for HSYA. However, in this study, propylene glycol dicaprylocaprate (PG) was selected as the oil phase rather than medium chain triglycerides and chuanxiong volatile oil, because PG can form a more stable w/o microemulsion, and the maximum water phase volume in this w/o microemulsion system is larger than the microemulsion formed by the other two oils. This study was undertaken to investigate the enhancing effect and mechanism of intestinal absorption for HSYA by using PG as the oil phase for the microemulsion.

\section{Materials and methods}

\section{Reagents and chemicals}

PG (Milgyol ${ }^{\circledR}$ 840) was kindly gifted by Sasol Chemical Co Ltd (Guangzhou, China). HSYA (84.2\% purity) was purchased from Changsha Keluoma Pharmaceutical Co Ltd (Changsha, China). Porcine pancreatic lipase was obtained from the Shanghai Chemical Reagent Co Ltd (Shanghai, China). Riboflavin (98.0\% purity) was purchased from Sigma-Aldrich Chemical Company (Shanghai, China). The lactate dehydrogenase diagnostic cassette used is a product of the Nanjing Jiancheng Bioengineering Institute (Nanjing, China). All other chemicals were of analytical grade and used as received.

\section{Animals}

Male Wistar rats (200-240 g), obtained from the Animal Center of the China Pharmaceutical University, were maintained under uniform experimental conditions (temperature $25 \pm 2{ }^{\circ} \mathrm{C}$, humidity $60 \pm 5 \%$, on a 12 -hour dark/light cycle) for 1 week prior to experiments. They were fed with a standard laboratory diet. All rats were fasted overnight before the experiment, with water ad libitum. All the animal experiments followed the recommendations of the Regulations for the Administration of Affairs Concerning Experimental Animals and were approved by the ethics committee of the China Pharmaceutical University.

\section{Preparation of HSYA w/o microemulsion}

The pseudoternary phase diagram was constructed to form the microemulsion. ${ }^{15}$ Cremophor RH40 (surfactant) and ethanol (cosurfactant) were mixed in a vial at room temperature followed by the addition of PG (oil phase), and then the mixture was blended for 5 minutes until they had completely mixed. The HSYA solution was then added to the mixture and stirred for 3 minutes until a clear and transparent w/o microemulsion was obtained.

\section{In vitro properties of HSYA w/o microemulsion}

The electrical conductivity $(\sigma)$ of the prepared microemulsion was determined at ambient temperature by a DS-11C conductivity meter coupled with a platinum electrode (Leici Instrument Co, Shanghai, China). Deionized water and saline were used to calibrate the instrument. The dynamic viscosity $(\eta)$ of the microemulsion was monitored using a rotational Brookfield DV-III viscometer (Brookfield Engineering Laboratories, Middleboro, MA). For measuring the particle size and zeta potential, the microemulsion was diluted 100 -fold by artificial intestinal juices. The particle size and zeta potential of the diluted microemulsion were determined by a Zetasizer 3000 instrument (Malvern Instruments, Worcestershire, UK).

\section{Oral bioavailability in bile duct-ligated and non-ligated rats}

Eighteen rats were divided into three groups. After anesthesia with $15 \%$ urethane $(1 \mathrm{~mL} / 100 \mathrm{~g})$, the first group (controls) underwent intraduodenal administration of HSYA solution $12.5 \mathrm{mg} / \mathrm{kg}$, the second group underwent intraduodenal administration of the microemulsion, and the third group underwent intraduodenal administration of the microemulsion after ligation of the bile duct. Bile duct ligation was performed as described previously, ${ }^{16}$ and the midline abdominal incision required was covered by sterile gauze to maintain body temperature. In brief, under anesthesia, the 
common bile duct was ligated with 3-0 silk and sectioned between the ligatures. The microemulsion was administered intraduodenally to the rats 2 hours after bile duct ligation, when all bile was depleted. The HSYA microemulsion dosage was $12.5 \mathrm{mg} / \mathrm{kg}$. After administration, serial blood samples were collected at 10, 20, 30, 45, 60, 90, 120, 180, 300, and 420 minutes. Each sample was immediately transferred to another heparinized tube and centrifuged at $5000 \mathrm{~g}$ for 5 minutes. The plasma was transferred to another tube and stored at $-20^{\circ} \mathrm{C}$ until analysis. For $100 \mu \mathrm{L}$ of plasma, $50 \mu \mathrm{L}$ of internal standard (IS) solution (riboflavin $10 \mu \mathrm{g} / \mathrm{mL}$ ) and $40 \mu \mathrm{L}$ of $6 \%$ perchloric acid were added. Each sample was vortex-mixed for 2 minutes and centrifuged for 10 minutes at $9000 \mathrm{~g}$. A $50 \mu \mathrm{L}$ aliquot of the supernatant was injected into the chromatography system. The pharmacokinetic parameters were calculated by statistical moment theory.

\section{Microemulsion lipolysis in vitro}

The lipolysis procedure was performed as described by Zangenberg et al, ${ }^{17,18}$ with some slight modification. In brief, $4 \mathrm{~g}$ of microemulsion was added to $20 \mathrm{~mL}$ of digestive buffer containing bile salt $5 \mathrm{mM}$, phosphatidylcholine $1 \mathrm{mM}$, trizma maleate $2 \mathrm{mM}, \mathrm{NaCl} 150 \mathrm{mM}$, and $\mathrm{CaCl}_{2} 20 \mathrm{mM}$. The solution was stirred gently and heated to $37^{\circ} \mathrm{C}$. Fresh pancreatic lipase extract was prepared by adding porcine pancreatic lipase to distilled water and stirring for 15 minutes. The supernatant was collected after centrifugation. Enzymatic digestion was started by adding the fresh pancreatic lipase extract to the previously mixed solution (1000 IU/mL lipase). The experiment was performed under continuous agitation by magnetic stirring $(100 \mathrm{rpm})$ and $\mathrm{pH}$ was maintained at 6.5 by titration with $\mathrm{NaOH} 0.05 \mathrm{M}$. After 30 minutes, 4-bromobenzeneboronic acid solution was added to halt lipase activity immediately. ${ }^{19}$ Microemulsion particle size after digestion was measured by the Zetasizer 3000 .

\section{Everted gut sac transport study}

Four grams of microemulsion were diluted 20-fold with Tyrode's solution. Another $4 \mathrm{~g}$ of microemulsion was first digested by pancreatic lipase, and then diluted to the same concentration as above with Tyrode's solution. HSYA in Tyrode's solution was used as the control.

The everted gut sacs were prepared using the jejuna from male Wistar rats. After fasting overnight, the rats were sacrificed under ether anesthesia, and the intestine extending from the pyloric end to the ileocecal junction was carefully excised. Fat and mesenteric attachments were removed. The separated segment was immediately chilled, and slowly flushed with ice-cold saline $0.9 \%$ using a syringe fitted with a blunt needle. Everted sacs $6 \mathrm{~cm}$ in length were made from the jejunum without Peyer's patches according to the method described by Wilson and Wiseman. ${ }^{20}$ The distal end of the sac was tied with a ligature. Another ligature was placed loosely around the proximal end.

The everted sacs were incubated in Tyrode's buffer $\left(37^{\circ} \mathrm{C}\right)$ saturated with $95 \% \mathrm{O}_{2}$ and $5 \% \mathrm{CO}_{2}$ in different donor solutions, and filled with $0.5 \mathrm{~mL}$ of blank Tyrode's buffer. At predetermined intervals $(15,30,45$, and 60 minutes $)$, $0.5 \mathrm{~mL}$ samples were taken from the internal medium, and $0.5 \mathrm{~mL}$ of fresh Tyrode's buffer $\left(37^{\circ} \mathrm{C}\right)$ was then added to the sacs. The samples were diluted by methanol followed by centrifugation, and $50 \mu \mathrm{L}$ of supernatant was then injected into a high-pressure liquid chromatography (HPLC) column to determine the concentration of HSYA. Cumulative transport (CT) was calculated by the following equation:

$$
\mathrm{CT}\left(\mu \mathrm{g} / \mathrm{cm}^{2}\right)=\frac{Q(\mu \mathrm{g})}{A\left(\mathrm{~cm}^{2}\right)}
$$

where $Q$ is the amount of cumulative drug molecule transport and $A$ is the area of the intestinal sac.

Intestinal tissue activity was measured for the duration of the experiment using the lactate dehydrogenase method. ${ }^{21}$ Liberation of cytosolic lactate dehydrogenase was determined in the incubation media in the absence and presence of the formulations using a lactate dehydrogenase diagnostic cassette.

\section{HPLC analysis of samples}

Chromatographic analysis was performed as previously described, ${ }^{22}$ with some modifications. A Shim-pack CLCODS C18 $(150 \times 4.6 \mathrm{~mm}$, internal diameter, $5 \mu \mathrm{m})$ HPLC column (Shimadzu, Kyoto, Japan) was used. The mobile phase comprised a potassium dihydrogen phosphate $0.022 \mathrm{~mol} / \mathrm{L}$ solution, adjusted to $\mathrm{pH} 3.0$ with phosphoric acid for pump A and acetonitrile $90 \%(\mathrm{v} / \mathrm{v})$ for pump B. The total flow rate was $1 \mathrm{~mL} / \mathrm{min}$. A gradient elution for A:B (0 minutes, $83: 17 ; 7$ minutes, $75: 25 ; 7.01$ minutes, stop) was used for the transport study. A gradient elution for A:B (0 minutes, 90:10; 20 minutes, 74:26; 20.01 minutes, stop) was used for the in vivo study. The column oven temperature was $30^{\circ} \mathrm{C}$. Detection was performed at a wavelength of $403 \mathrm{~nm}$.

\section{Statistical analysis}

All values are expressed as the mean \pm standard deviation. One-way analysis of variance and Student's $t$-test were used to analyze the results, with $P<0.05$ accepted as the minimum level of significance. 


\section{Results}

\section{Optimization of microemulsion composition}

A typical pseudoternary phase diagram of PG, Cremophor RH40/ethanol (w/w, 2:1) and water was constructed. As shown in Figure 1, a clear and transparent microemulsion was identified in the microemulsion zone. The optimal microemulsion was selected from this area. w/o microemulsion composed of $30 \%$ PG, $46 \%$ Cremophor RH40/ethanol, and $24 \%$ water is represented by a black triangle. The HSYA drug loading was $3.12 \mathrm{mg} / \mathrm{g}$.

\section{Properties of microemulsion in vitro}

The electrical conductivity, viscosity, particle size, polydispersity, and zeta potential of the diluted w/o microemulsion are summarized in Table 1. The electrical conductivity of the microemulsion was $38.0 \mu \mathrm{S} / \mathrm{cm}$, viscosity was $567.7 \pm 5.3 \mathrm{cP}$, diluted particle size was about $144.2 \mathrm{~nm}$, and polydispersity was approximately 0.6 . However, the zeta potential of the diluted microemulsion was positive $(24.6 \pm 1.5 \mathrm{mV})$.

\section{Oral bioavailability in bile duct-ligated and non-ligated rats}

The HSYA plasma concentration-time (AUC) profiles after intraduodenal administration of microemulsion to bile ductnonligated rats, bile duct-ligated rats, and the control solution are shown in Figure 2, and the main pharmacokinetic parameters are listed in Table 2. The time to peak plasma concentration and $\mathrm{AUC}_{0-\infty}$ values for HSYA after intraduodenal administration of the microemulsion to bile ductnonligated rats were significantly higher than that for bile duct-ligated rats $(P<0.01)$. The relative bioavailability after intraduodenal administration of microemulsion to bile duct-nonligated and bile duct-ligated rats was 1937\% and $181 \%$, respectively. These results show that the enhanced absorption ability of the microemulsion was significantly affected by secretion of bile.

\section{Lipolysis of w/o microemulsion in vitro}

Consumption of $\mathrm{NaOH}$ during the study, reflecting the extent of lipolysis, is shown in Figure 3. The values presented have been corrected by subtracting the amount of $\mathrm{NaOH}$ consumed when the experiment was carried out without any formulation present. After lipolysis, the microemulsion was clear and transparent. The mean particle size after lipolysis was $53.3 \mathrm{~nm}$.

\section{HSYA transport from microemulsion using everted gut sacs}

Figure 4 shows the time profiles for cumulative intestinal permeation of HSYA for the diluted microemulsion, digested microemulsion, and control solution. Cumulative intestinal HSYA permeation of the diluted microemulsion was not significantly different from that of the control solution.

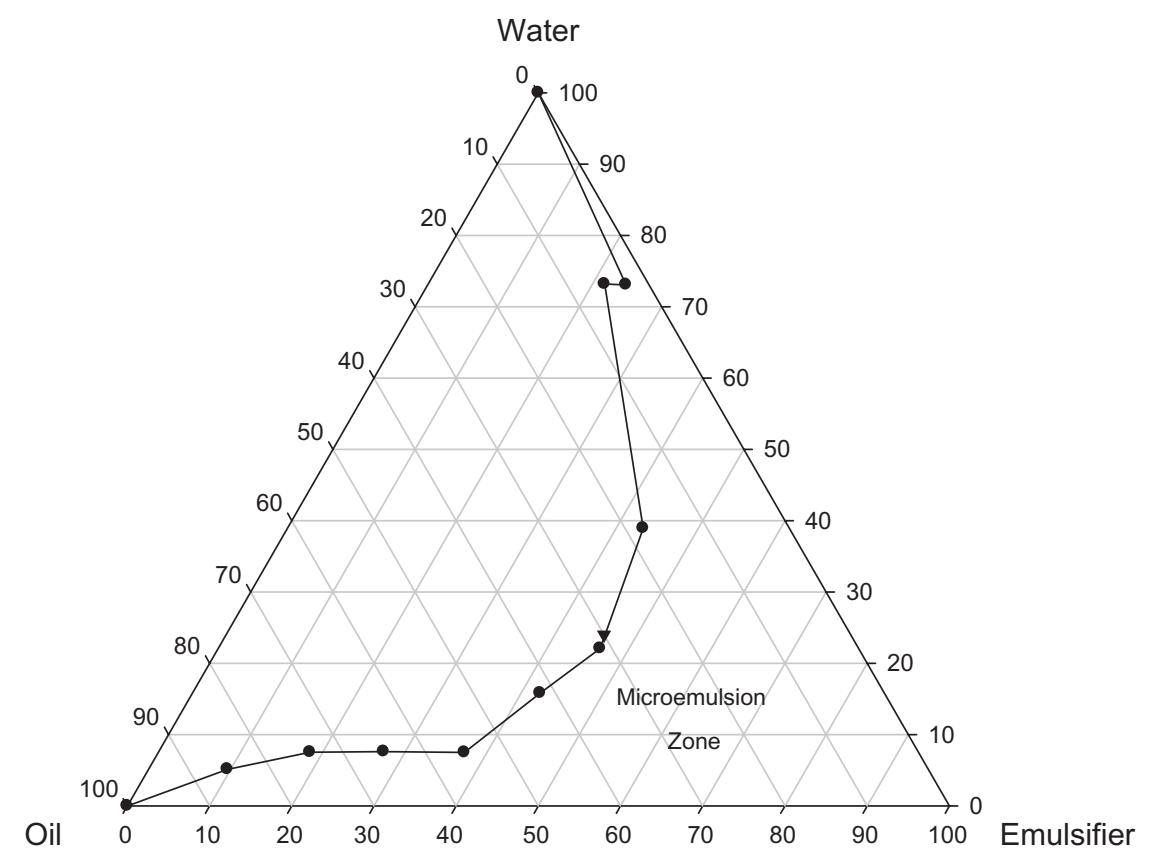

Figure I Pseudoternary phase diagram of the system. Clear and transparent microemulsions were formed in the W/O area. Oil was PG and emulsifier was mixture of Cremophor RH40 and ethanol (2:1). 
Table I In vitro properties of the w/o microemulsion and diluted microemulsion

\begin{tabular}{lllll}
\hline W/O microemulsion & \multicolumn{3}{l}{ Diluted microemulsion } \\
\hline$\sigma(\mu \mathrm{S} / \mathrm{cm})$ & $\eta(\mathrm{cP})$ & Size $(\mathrm{nm})$ & Polydispersity & $\begin{array}{l}\text { Zeta } \\
\text { potential } \\
(\mathrm{mV})\end{array}$ \\
\hline $38.0 \pm 2.5$ & $567.7 \pm 5.3$ & $144.2 \pm 10.5$ & $0.60 \pm 0.1 \mathrm{I}$ & $24.6 \pm 1.5$ \\
\hline
\end{tabular}

However, at 60 minutes, cumulative intestinal HSYA permeation of the digested microemulsion was 5.56-fold higher than that of the diluted microemulsion.

\section{Discussion}

A microemulsion is a thermodynamically stable, clear, transparent system and shows a broad range of structures. Depending on the spontaneous curvature and flexibility of the surfactant monolayer, bicontinuous microemulsion and liquid crystals appear during formation of a microemulsion. ${ }^{23}$ In our research, in order to achieve the optimal water content, formulations with higher viscosity and electrical conductivity were selected, which could be near to the structure of bicontinuous microemulsion. The size and zeta potential of the diluted microemulsion were determined to characterize its properties after entering the gastrointestinal tract via oral administration. The results show that the size of the microemulsion was small, while the zeta potential was positive. The high positive charge may contribute to its high intestinal absorption. ${ }^{24}$ The w/o microemulsion could be transformed to a $\mathrm{o} / \mathrm{w}$ emulsion or a $\mathrm{w} / \mathrm{o} / \mathrm{w}$ multiemulsion after it is diluted.

Microemulsion systems with nonionic surfactants, good biological acceptance, and emulsifying ability are used extensively. ${ }^{25}$ Microemulsions have been widely used in improving the bioavailability of insoluble drugs ${ }^{26}$ and proteins. ${ }^{7}$ In our study, the w/o microemulsion significantly increased the oral bioavailability of HSYA, a BCS III drug. We postulate there are two reasons for the absorption-enhancing

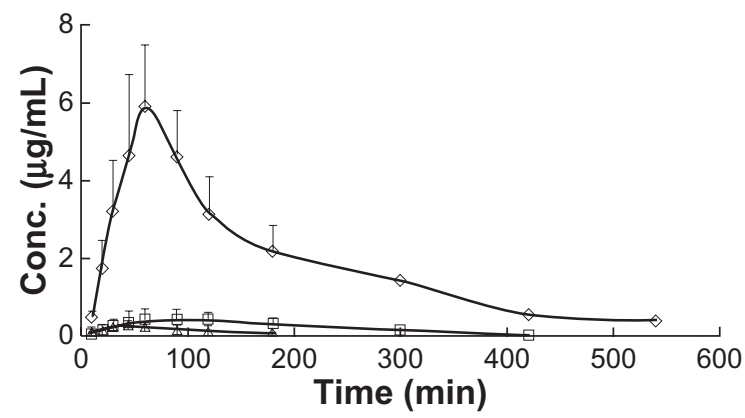

Figure 2 Plasma concentration-time profiles of HSYA after intraduodenal (id) administration of microemulsion to bile duct non-ligated rats $(\diamond)$, bile duct ligated rats $(\square)$ and id of control solution $(\triangle)(n=6)$.
Table 2 Pharmacokinetics parameters of HSYA after intraduodenal (id) administration of w/o microemulsion to bile duct nonligated rats, bile duct ligated rats and id of control solution $(n=6)$

\begin{tabular}{llll}
\hline & Nonligated & Ligated & Control \\
\hline $\mathrm{C}_{\max }(\mu \mathrm{g} / \mathrm{ml})$ & $5.90 \pm 1.59 * * \ldots$ & $0.49 \pm 0.25$ & $0.35 \pm 0.18$ \\
$\mathrm{MRT}(\mathrm{min})$ & $166.88 \pm 16.85^{* *}$ & $150.98 \pm 16.46^{* *}$ & $81.44 \pm 13.66$ \\
$\mathrm{AUC}_{0-\infty}$ & $980.55 \pm 317.55^{* *, \ldots}$ & $91.62 \pm 38.67 *$ & $50.63 \pm 16.68$ \\
$(\mu \mathrm{g} / \mathrm{m} / \mathrm{min})$ & & & \\
$\mathrm{F}_{\text {rel }}(\%)$ & 1937 & 181 & 100 \\
\hline
\end{tabular}

Notes: $* P<0.05$ compared with control; **P $<0.01$ compared with control; $\# P<0.01$ Non-ligated group compared with ligated group.

Abbreviations: $M R T$, mean retention time; $F_{\text {rel }}$ relative bioavailability.

effect of microemulsions. Firstly, the lipids and surfactants in the microemulsion can increase cell membrane fluidity and open the tight junctions between cells to improve the permeability of hydrophilic drug molecules. ${ }^{27,28}$ Secondly, because of intercalation of the digestive products of exogenous lipids into the intestinal bile salt-endogenous phospholipids mixed micelles, the amount of HSYA partitioned into the micelles increases, leading to an increase in the solubilization capacity of the gastrointestinal tract. Furthermore, our study demonstrated the solubility of HSYA in a mixture of medium-chain fatty acids and propylene glycol (digestive products of PG, $2423.26 \mu \mathrm{g} / \mathrm{mg}$ ). Therefore, when microemulsion is applied, HSYA could be dissolved in mixed micelles formed by enzymatic products of oil, bile salts, and phospholipids, and then directly enter the blood circulation by vesicle-mediated uptake or collision transfer. ${ }^{5,29,30}$

Lipid-based formulations are digested by pancreatic lipase, and the rate and extent of lipolysis greatly affects the oral bioavailability of poorly soluble drugs. ${ }^{31}$ After digestion, triglycerides are converted to diglycerides, monoglycerides, and fatty acids, ${ }^{5}$ and, similarly, propylene glycol diesters are converted to propylene glycol monoesters and fatty acids. It has been found that fatty acids can increase the permeability of poorly absorbed drugs. ${ }^{32}$ However, our study showed that the oral bioavailability in bile duct-nonligated rats was higher than that in the bile duct-ligated rats. Bile salts and

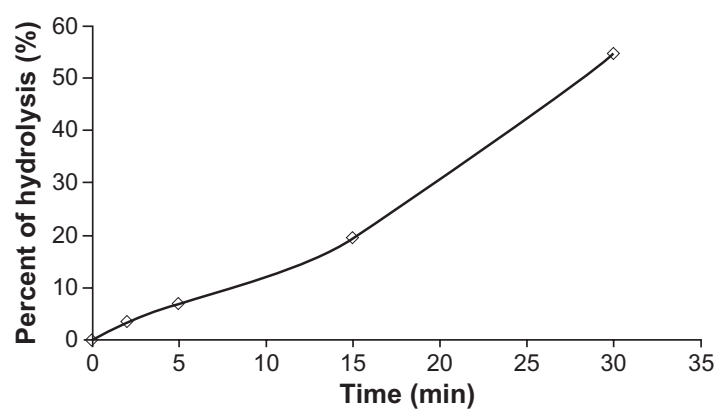

Figure 3 The percent of lipolysis-time profile of the microemulsion. 


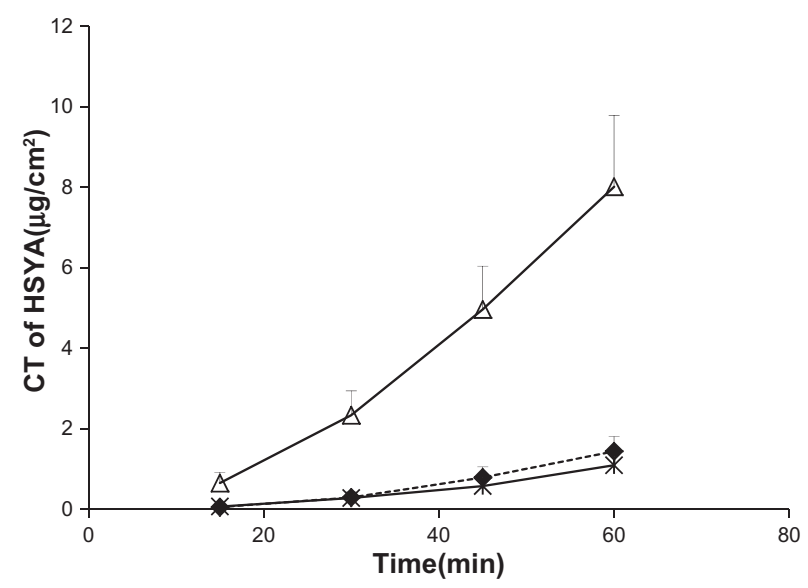

Figure 4 The cumulative transport of HSYA after administration of digested microemulsion $(\triangle)$, diluted microemuslion $(\triangleleft)$ and control solution $(\times)$.

pancreatic lipase were secreted by the bile duct, indicating that digestion of the microemulsion is also important for enhanced absorption of hydrophilic drugs. In addition, the particle size in the digested microemulsion was very small $(53.3 \mathrm{~nm})$, which was also beneficial for drug absorption. Importantly, most studies have suggested that the best absorption enhancing effect appears when drug molecules and enhancers arrive at the absorption sites at the same time. ${ }^{33}$ After lipolysis, the microemulsion was still uniform, even after centrifugation at $12,000 \mathrm{~g}$. Therefore, the HSYA and digestive products can arrive simultaneously at the absorption sites after digestion.

In order to illustrate further the mechanism of the enhancing effect of microemulsions, the everted gut sac model was used to study the permeability of the microemulsion and the digested microemulsion. The everted gut sac is a popular model for the investigation of intestinal permeability. Through pre-experiments, a 20 -fold diluted microemulsion was selected as the donor solution to ensure activity of the intestinal gut sac, with activity detected using the lactate dehydrogenase method. ${ }^{21}$ The results demonstrated that the permeability of the diluted microemulsion was similar to that of the control solution, while the permeability of the digested microemulsion was far higher than that of the control solution. This again suggests that the digested microemulsion can enhance absorption more effectively.

\section{Conclusion}

In conclusion, HSYA could be effectively incorporated into a microemulsion system consisting of PG, Cremophor RH40/ ethanol, and water. The physicochemical characteristics of the microemulsion have been described. The microemulsion can markedly improve the oral bioavailability of HSYA.
Furthermore, digestion of the microemulsion by pancreatic lipase enhances the oral bioavailability of water-soluble drugs.

\section{Acknowledgments}

This study was supported by the key program 30430790 of the State Nature and Science Foundation and the major project (No. 2009ZX09310-004 and 2009ZX09103-344) of the National Science and Technology of China for New Drug Development.

\section{Disclosure}

The authors report no conflicts of interest in this work.

\section{References}

1. Hauss DJ. Oral lipid-based formulations. Adv Drug Deliv Rev. 2007; 59(7):667-676.

2. Piao H, Kamiya N, Watanabe J, et al. Oral delivery of diclofenac sodium using a novel solid-in-oil suspension. Int J Pharm. 2006;313(1-2): 159-162.

3. Wu W, Wang Y, Que L. Enhanced bioavailability of silymarin by self-microemulsifying drug delivery system. Eur J Pharm Biopharm. 2006;63(3):288-294.

4. Strickley RG. Solubilizing excipients in oral and injectable formulations. Pharm Res. 2004;21(2):201-230.

5. Porter CJ, Trevaskis NL, Charman WN. Lipids and lipid-based formulations: Optimizing the oral delivery of lipophilic drugs. Nat Rev Drug Discov. 2007;6(3):231-248.

6. Khoo SM, Shackleford DM, Porter CJ, Edwards GA, Charman WN. Intestinal lymphatic transport of halofantrine occurs after oral administration of a unit-dose lipid-based formulation to fasted dogs. Pharm Res. 2003;20(9):1460-1465.

7. Cheng MB, Wang JC, Li YH, et al. Characterization of water-in-oil microemulsion for oral delivery of earthworm fibrinolytic enzyme. J Control Release. 2008;129(1):41-48.

8. Prasad YV, Puthli SP, Eaimtrakarn S, et al. Enhanced intestinal absorption of vancomycin with Labrasol and D-alpha-tocopheryl PEG 1000 succinate in rats. Int J Pharm. 2003;250(1):181-190.

9. Constantinides PP. Lipid microemulsions for improving drug dissolution and oral absorption: Physical and biopharmaceutical aspects. Pharm Res. 1995;12(11):1561-1572.

10. Liu YN, Zhou ZM, Chen P. Evidence that hydroxysafflor yellow A protects the heart against ischaemia-reperfusion injury by inhibiting mitochondrial permeability transition pore opening. Clin Exp Pharmacol Physiol. 2008;35(2):211-216.

11. Zhu H, Wang Z, Ma C, et al. Neuroprotective effects of hydroxysafflor yellow A: In vivo and in vitro studies. Planta Med. 2003;69(5): 429-433.

12. Zhu HB, Wang ZH, Tian JW, Fu FH, Liu K, Li CL. Protective effect of hydroxy safflor yellow A on experimental cerebral ischemia in rats. Yao Xие Хие Bao. 2005;40(12):1144-1146. Chinese.

13. Wang S, Sun M, Ping Q. Enhancing effect of Labrafac Lipophile WL 1349 on oral bioavailability of hydroxysafflor yellow A in rats. Int J Pharm. 2008;358(1-2):198-204.

14. Qi J, Sun M, Ping Q, et al. The mechanisms for enhanced oral absorption of hydroxysafflor yellow A by Chuanxiong volatile oil. Planta Med. 2010;76(8):786-792.

15. Aboofazeli R, Lawrence MJ. Investigations into the formation and characterization of phospholipid microemulsions. I Pseudo-ternary phase diagrams of systems containing water-lecithin-alcohol-isopropyl myristate. Int J Pharm. 1993;93(1-3):161-175. 
16. Tavakoli S, Hajrasouliha AR, Jabehdar-Maralani $\mathrm{P}$, et al. Modulated hemodynamic response to clonidine in bile duct-ligated rats: The role of nitric oxide. Eur J Pharmacol. 2006;542(1-3):148-153.

17. Zangenberg NH, Mullertz A, Kristensen HG, Hovgaard L. A dynamic in vitro lipolysis model. I. Controlling the rate of lipolysis by continuous addition of calcium. Eur J Pharm Sci. 2001;14(2):115-122.

18. Zangenberg NH, Mullertz A, Kristensen HG, Hovgaard L. A dynamic in vitro lipolysis model. II: Evaluation of the model. Eur J Pharm Sci. 2001;14(3):237-244.

19. Fatouros DG, Bergenstahl B, Mullertz A. Morphological observations on a lipid-based drug delivery system during in vitro digestion. Eur $J$ Pharm Sci. 2007;31(2):85-94.

20. Wilson TH, Wiseman G. The use of sacs of everted small intestine for the study of the transference of substances from the mucosal to the serosal surface. J Physiol. 1954;123(1):116-25.

21. Cornaire G, Woodley J, Hermann P, Cloarec A, Arellano C, Houin G. Impact of excipients on the absorption of P-glycoprotein substrates in vitro and in vivo. Int J Pharm. 2004;278(1):119-131.

22. Qi J, Jin X, Huang L, Ping Q. Simultaneous determination of hydroxysafflor yellow $\mathrm{A}$ and ferulic acid in rat plasma after oral administration of the co-extractum of Rhizoma chuanxiong and Flos Carthami by HPLCdiode array detector. Biomed Chromatogr. 2007;21(8):816-822.

23. Formariz TP, Chiavacci LA, Sarmento VH, et al. Structural changes of biocompatible neutral microemulsions stabilized by mixed surfactant containing soya phosphatidylcholine and their relationship with doxorubicin release. Colloids Surf B Biointerfaces. 2008;63(2):287-295.

24. Yang SC, Benita S. Enhanced absorption and drug targeting by positively charged submicron emulsions. Drug Develop Res. 2010;50:476-486.
25. Alany RG, Rades T, Agatonovic-Kustrin S, Davies NM, Tucker IG. Effects of alcohols and diols on the phase behaviour of quaternary systems. Int J Pharm. 2000;196(2):141-145.

26. Kawakami K, Yoshikawa T, Hayashi T, Nishihara Y, Masuda K. Microemulsion formulation for enhanced absorption of poorly soluble drugs. II. In vivo study. J Control Release. 2002;81(1-2):75-82.

27. Sha X, Yan G, Wu Y, Li J, Fang X. Effect of self-microemulsifying drug delivery systems containing Labrasol on tight junctions in Caco-2 cells. Eur J Pharm Sci. 2005;24(5):477-486.

28. Yeh PY, Smith PL, Ellens H. Effect of medium-chain glycerides on physiological properties of rabbit intestinal epithelium in vitro. Pharm Res. 1994;11(8):1148-1154.

29. Shiau YF, Kelemen RJ, Reed MA. Acidic mucin layer facilitates micelle dissociation and fatty acid diffusion. Am J Physiol. 1990;259(4 Pt 1): G671-G675.

30. Thomson AB, Schoeller C, Keelan M, Smith L, Clandinin MT. Lipid absorption: Passing through the unstirred layers, brush-border membrane, and beyond. Can J Physiol Pharmacol. 1993;71(8):531-555.

31. Dahan A, Hoffman A. The effect of different lipid based formulations on the oral absorption of lipophilic drugs: The ability of in vitro lipolysis and consecutive ex vivo intestinal permeability data to predict in vivo bioavailability in rats. Eur J Pharm Biopharm. 2007;67(1):96-105.

32. Kimura Y, Hosoda Y, Shima M, Adachi S, Matsuno R. Physico-chemical properties of fatty acids for assessing the threshold concentration to enhance the absorption of a hydrophilic substance. Biosci Biotechnol Biochem. 1998;62(3):443-447.

33. Aungst BJ. Intestinal permeation enhancers. J Pharm Sci. 2000;89(4): 429-442.
International Journal of Nanomedicine

\section{Publish your work in this journal}

The International Journal of Nanomedicine is an international, peerreviewed journal focusing on the application of nanotechnology in diagnostics, therapeutics, and drug delivery systems throughout the biomedical field. This journal is indexed on PubMed Central,

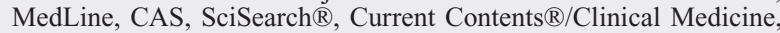

\section{Dovepress}

Journal Citation Reports/Science Edition, EMBase, Scopus and the Elsevier Bibliographic databases. The manuscript management system is completely online and includes a very quick and fair peer-review system, which is all easy to use. Visit http://www.dovepress.com/ testimonials.php to read real quotes from published authors. 